Full Paper

\title{
Veränderungen in der Reifeentwicklung der Trauben im Südtiroler Weinbau laut den Daten der jährlichen Reifetests von 1985 bis heute
}

\author{
Changes in the ripening development of grapes in South Tyrolean viticulture according to the data of \\ the annual ripeningtests from 1985 up to now
}

Cambiamenti nello sviluppo della maturazione dell'uva in Alto Adige secondo i dati dei test di maturazione annuali dal 1985 ad oggi

Barbara Raifer ${ }^{1}$, Andreas Sölva ${ }^{1}$, Eva Überegger ${ }^{1}$, Christoph Sanoll ${ }^{1}$, Josef Terleth ${ }^{1}$, Florian Haas ${ }^{1}$

1 Versuchszentrum Laimburg, Pfatten, Italien

\section{ABSTRACT}

Since 1985, the Laimburg Research Centre has been carrying out, on on a weekly (a few weeks before full ripening), a test on the most important South Tyrolean grape for the purpose of monitoring the ripening status and help winegrowers to plan their harvest. The resulting data represent at the same time an interesting documentation of the changes which occurred during this period. In addition to climate changes, with increasing average temperatures, the last three and a half decades in South Tyrol have been characterized by major changes in viticulture and, in particular, by the pursuit of higher grape and wine quality. Today we register the same grape ripeness stage 19 to 50 days earlier than in 1985; the majority of cases has in average an about 3-weeks earlier ripening. It is not possible to determine from the available data to what extent these changes are due to the changing climatic conditions or changes in cultivation. However, the latter might also play a role, especially in cases with an above-average early ripening.

At the same sugar content, the total acidity of musts did not decrease; likewise, the $\mathrm{pH}$ values did not increase. This should have been expected due to the warmer conditions during the ripening period of the grapes. Rather, data show that, for the same sugar content, the total acidity values have either remained similar or are higher today than in the past. In some cases, with the same total acidity, the $\mathrm{pH}$ values are also lower today than in the past.

\section{KEYWORDS}

grapevine, ripening development, climate change, sugar, acidity

\section{CITE ARTICLE AS}

Raifer Barbara, Sölva Andreas, Überegger Eva et.al. (2021).

Changes in the ripening development of grapes in South Tyrolean viticulture according to the data of the annual ripeningtests from 1985 up to now. Laimburg Journal 03/2021 DOI: $10.23796 /$ LJ/2021.003

\section{CORRESPONDING AUTHOR}

Barbara Raifer Laimburg 6, Pfatten, 39040 Auer, Italien

barbara.raifer@laimburg.it 00390471969616 


\section{EINLEITUNG}

Die zeitlichen Veränderungen in der phänologischen Entwicklung der Reben, der frühere Austrieb, die um zwei bis drei Wochen frühere Blüte und auch der frühere Reifebeginn und Erntezeitpunkt lassen keinen Zweifel aufkommen, das Klima hat sich bereits deutlich verändert [1] [2] [3] [4]. Wir ernten heute Trauben mit höheren Zuckergehalten, die Gesamtsäuregehalte sind niedriger, oft so niedrig, dass ein Zusatz von Säure notwendig wird. Der pH-Wert der Moste ist angestiegen, der Charakter der Weine hat sich verändert. Dies nicht immer zum Negativen. In vielen Weinbaugebieten, so z. B. auch für das Bordeaux kann belegt werden, dass sich das allgemeine Qualitätsniveau erhöht hat und heute deutlich mehr gute bis sehr gute Weinjahrgänge pro Jahrzehnt zu verzeichnen sind [5]. Dies trifft auch für Südtirol [6] [7] teilweise zu. Auch in Südtirol hat sich der Weinbau in den letzten 3-4 Jahrzehnten positiv entwickelt und dies ist nicht zuletzt auf die inzwischen deutlich höhere Reifeentwicklung der Trauben, zurückzuführen. Neben diesen erfreulichen Entwicklungen zeichnen sich für den Weinbau in Zukunft leider auch weniger erfreuliche Veränderungen infolge der ansteigenden Temperaturen ab [8] [9].

In der Zeit vor der Lese werden am Versuchszentrum Laimburg regelmäßig jeden Montag bei allen wichtigen Rebsorten in mehreren Standorten repräsentative Beerenproben entnommen und im Labor für Wein- und Getränkeanalysen analysiert. Begonnen wurde mit diesen Reifetests im Jahre 1985, einige Lagen und Sorten kamen allerdings erst später dazu. Die durchgeführten Mostanalysen sollten eine Hilfe für die Südtiroler Kellereien sein, zeitgerecht Hinweise zum Reifeverlauf des Jahrganges liefern und dadurch die Planung der Ernte erleichtern. Bei der Auswahl der Anlagen und der Planung der Arbeiten im Jahre 1985 dachte man nicht daran, dass diese Daten auch eine Dokumentation der Entwicklung des Weinbaues sein würden, in einer Zeit weitreichender und einschneidender Veränderungen. Veränderungen, die auf den Klimawandel zurückzuführen sind, auf den in Südtirol erfolgten Temperaturanstieg von $1,8^{\circ} \mathrm{C}$ im Zeitraum von $1970-2018$ [10], aber auch auf die bewusst gesetzten Veränderungen im Anbau, im Bestreben die Weinqualität zu verbessern. Hätte man im Blick gehabt, mit diesen Daten zugleich auch die Entwicklung dokumentieren $\mathrm{zu}$ wollen, hätte z.B. allein die jährliche Erhebung einiger Zusatzinformationen einiges mehr an Information und Aussagen erlaubt. Dem war nicht so und daher stößt man bei der Auswertung der Daten auch schnell an Grenzen. Aber auch so erlauben die Reifetestdaten noch interessante Einblicke und bezeugen, wie deutlich sich die Realitäten im Südtiroler Weinbau in den letzten Jahrzehnten verändert haben.

In den letzten 35 Jahren wurden in Südtirol die meisten Anlagen erneuert und außer bei der Sorte Vernatsch wurde dabei in den meisten Fällen von Perglerziehung auf Spalier umgestellt. Für die Entnahme der Reifetests wurde daher, bei Rodung der bisherigen Reifetest-Anlage, zwangsläufig auf eine jüngere Anlage ausgewichen, meist auf eine Anlage in der Nähe oder zumindest auf eine Rebanlage in einem vergleichbaren Standort. Zur Erneuerung vieler Anlagen und dem Wechsel des Erziehungssystems, kamen im Laufe der letzten 35 Jahre weitere Veränderungen im Anbau hinzu. In den allermeisten Fällen wurden die durchschnittlichen Erträge deutlich abgesenkt von ursprünglichen Mengen zwischen 1-2 oder auch 2,5 kg Trauben pro $\mathrm{m}^{2}$ in den 80er und den ersten $90 \mathrm{er}$ Jahren auf heute $0,5-1 \mathrm{~kg}$ Trauben pro $\mathrm{m}^{2}$ Standraum der Reben. Eine Ausnahme stellt dabei die Sorte Vernatsch dar, bei der die Erträge bis jetzt meist zwischen 1,2-1,5 kg Trauben pro $\mathrm{m}^{2}$ Standraum der Reben liegen. Auch die Anlagen in den Tallagen weisen meist eher etwas höhere Erträge auf. Erst in allerjüngster Zeit zeichnet sich auch bei Vernatsch, neben dem weiter anhaltenden Rückgang der Anbaufläche, eine teilweise Hinwendung zu niedrigeren Erträgen und höherem Qualitätsniveau ab. Die überwiegend niedrigen Erträge bei allen anderen Sorten, werden seit Jahren mit sehr mäßiger Erhaltungsdüngung erzielt. Aufgrund der in früheren Jahrzehnten sehr hohen Düngergaben wurde in den letzten Jahrzehnten vielfach die Düngung über Jahre vollständig ausgesetzt und erst bei bestätigter niedriger Versorgung aufgrund von Bodenanalysen oder bei zu starkem Rückgang des vegetativen Wachstums, in Maßen wieder aufgenommen [11].

Außerdem war in Südtirol im Jahre 1985 ein Großteil der Rebanlagen nicht mit einem Bewässerungssystem ausgestattet. Trockenschäden waren bis dahin die große Ausnahme. Erst nach den Trockenjahren Anfangs der 90er Jahre änderte sich dies schnell. Innerhalb weniger Jahre wurden über $90 \%$ der Rebanlagen Südtirols mit einer fix eingebauten Bewässerungsanlage und entsprechendem Zugang zu Bewässerungswasser ausgestattet.

Essigfäule kam Mitte bis Ende der 80er Jahre des letzten Jahrhunderts auf. Das Phänomen war bis dahin so gut wie unbekannt und löste die gefürchtete Traubenfäule oder Botrytis weitgehend ab. Die Ertragsausfälle waren bei den Sorten unterschiedlich hoch, vor allem bei den Burgundersorten aber enorm. Bald erkannte man, dass lockerbeerige Trauben von diesem Problem größtenteils verschont blieben, während kompakte Trauben von Essigfäule vollständig zerstört wurden [12]. Die Förderung der Lockerbeerigkeit, zunächst händisch, durch frühes Entblättern während der Blüte [13], durch Abstreifen der Trauben und durch Traubenhalbieren, kam auf. Chemisches Ausdünnen wurde in Versuchen ausgereizt, blieb in Südtirol aber auf den Einsatz von Gibberellin bei einigen Sorten, vor allem den BurgunderSorten, beschränkt. Maschinelle Förderung der Lockerbeerigkeit wurde in mehreren Varianten geprüft, aber erstmals mit dem Gerät zur pneumatischen Entblätterung, welches beim Einsatz zur Blüte oder unmittelbar danach auch Beeren zum Ausfallen bringt, erfolgreich etabliert. Erst Jahre später kamen die Bürsten dazu. Schließlich lösten lockerbeerige Klone bei einigen Sorten das Problem grundlegend [14] [15]. Erst nachdem man bereits mehrere Möglichkeiten zur Förderung der Lockerbeerigkeit in der Praxis etabliert hatte, wurde klar, dass es sich dabei um eine erste Anpassung des Weinbaues an den Klimawandel gehandelt hat. Die wärmeren Temperaturen in der Zeit der Traubenreife hemmten die Entwicklung des Botrytispilzes, der klassischen Traubenfäule, förderten aber bei kleinsten Beschädigungen der Beeren die Bildung von Essigsäure. Die Klimaänderung hatte sich bemerkbar gemacht.

All diese Veränderungen hielten in den letzten dreieinhalb Jahrzehnten im Südtiroler Weinbau verbreitet Einzug. Abzugrenzen wie sich diese Maßnahmen im Einzelnen ausgewirkt haben und wieviel der inzwischen erkennbaren Veränderungen der Reifeentwicklung auf die Klimabedingungen zurückgeführt werden kann, ist anhand der hier vorliegenden Daten kaum möglich. Dies ist in vorliegender Arbeit auch nicht das primäre Ziel. Vielmehr geht es hier darum, aufzuzeigen wie sich die Reifeentwicklung verändert hat, die der wichtigsten Reifeparameter und auch die zeitliche Entwicklung der Reife. 
Die hier dargestellten Daten erfassen die letzte Reifeentwicklungsphase der jeweiligen Sorten und Standorte. Vielfach wurden in den Tagen nach dem letzten Reifetesttermin die Trauben der jeweiligen Anlage gelesen. Es ist aber nicht immer so, dass die Reifeentwicklung bis zur Lese dokumentiert wurde. Es kann sein, dass in einzelnen Fällen erst ein, zwei oder im Extremfall auch drei Wochen nach dem letzten Reifetest geerntet wurde.

\section{METHODE}

Die wichtigsten Informationen zu den Testanlagen deren Daten nachfolgend dargestellt werden, sind in Tabelle 1 zusammengefasst.

\section{DIE ENTNAHME DER REIFETESTS}

Die Beeren für die Analysen wurden jeden Montag in den letzten 5-6 Wochen vor der voraussichtlichen Ernte entnommen, unabhängig von der Witterung. Feuchte Beeren, infolge von Niederschlägen, wurden im Labor mit Küchenpapier getrocknet. Für die Entnahme der Proben wurden 300 Beeren pro Anlage so gesammelt, dass dabei weitestgehend alle Teile der Anlage vertreten waren. Es wurden mehrere Reihen der Anlage ganz abgeschritten und dabei gleichmäßig verteilt, von zufällig ausgewählten Trauben, Beeren entnommen. Pro Traube wurden drei Beeren entnommen, jeweils eine im oberen, mittleren und unteren Teil.

\section{DIE AUFBEREITUNG DER PROBEN UND ANALYSEN}

Diese Beerenproben wurden manuell gequetscht, mittels einer kleinen Labormembranpresse zweimal innerhalb 1 Min. bei 2 bar gepresst, der Most zentrifugiert (HERM-

Tab. 1: Übersicht der Rebanlagen in denen die Beerenproben für die Reifetest entnommen wurden. // Overview of the vineyards where the berry samples for the ripening test were taken.

\begin{tabular}{|c|c|c|c|c|}
\hline $\begin{array}{l}\text { Sorte } \\
\text { Variety }\end{array}$ & $\begin{array}{l}\text { Anlage } \\
\text { Site }\end{array}$ & $\begin{array}{l}\text { Meereshöhe ( } m \text { ü. NN) } \\
\text { Sea level ( } m \text { a.s.l) }\end{array}$ & $\begin{array}{l}\text { Zeitraum } \\
\text { Period }\end{array}$ & $\begin{array}{l}\text { Erziehungsform } \\
\text { Training system }\end{array}$ \\
\hline Weißburgunder & Eppan-Berg & 560 & 1987-2004 & Pergl \\
\hline Weißburgunder & Eppan-Berg & 570 & $2005-2019$ & Spalier \\
\hline Weißburgunder & Terlan-Eyrl & 280 & $1985-2013$ & Pergl \\
\hline Weißburgunder & Terlan-Eyrl & 280 & 2014-2019 & Spalier \\
\hline Blauburgunder & Kastelbell-Fleck & 600 & $1988-2019$ & Spalier \\
\hline Blauburgunder & Neumarkt-Mazzon & 360 & $1992-2002$ & Pergl \\
\hline Blauburgunder & Neumarkt-Mazzon & 350 & 2003-2019 & Spalier \\
\hline Chardonnay & Kurtinig-Dorf & 200 & 1986-2004 & Pergl \\
\hline Chardonnay & Kurtinig-Giatl & 200 & 2005-2017 & Pergl \\
\hline Ruländer & Kurtinig-Moos & 200 & 1986-2019 & Pergl \\
\hline Ruländer & Salurn-Rosslauf & 200 & $1986-2002$ & Pergl \\
\hline Ruländer & Salurn-Pfatten & 200 & 1993-2019 & Pergl \\
\hline Gewürztraminer & Tramin Rungg & 330 & $1985-2002$ & Spalier \\
\hline Gewürztraminer & Tramin Rungg & 330 & 2003-2019 & Spalier \\
\hline Sauvignon & Terlan-Winkl & 270 & 1990-2019 & Spalier \\
\hline Sauvignon & Eppan-Naun & 400 & 1993-2012 & Spalier \\
\hline Sauvignon & Eppan-Naun & 400 & 2012-2019 & Spalier \\
\hline Vernatsch & Marling-Schickenburg & 380 & $1985-2019$ & Pergl \\
\hline Vernatsch & Eppan-Kreit & 450 & $1985-2019$ & Pergl \\
\hline Vernatsch & Kaltern-Barleit & 370 & $1985-2019$ & Pergl \\
\hline Vernatsch & Kaltern-See & 230 & $1985-2019$ & Pergl \\
\hline Vernatsch & Bozen-St. Magdalena & 300 & 1986-2019 & Pergl \\
\hline
\end{tabular}


LE Z513; 3500 Umdrehungen in 5 Min.) und filtriert (Faltenfilter Macherey Nagel 619 G $1 / 4 \bullet \varnothing 150 \mathrm{~mm}$ ). Es erfolgten Messungen des Mostgewichtes ( ${ }^{\circ} \mathrm{KMW}$ ) (refraktometrisch), der Gesamtsäure, ausgedrückt in Weinsäure (g/L) (titrimetrisch), des pH-Wertes (potenziometrisch), der Apfelsäure (enzymatisch), Weinsäure (colorimetrisch) und des hefeverwertbaren Stickstoffs (HVS) (enzymatisch).

Im Laufe der Zeit wurde die Analytik weiterentwickelt und seit nunmehr ca. 10 Jahren werden die Beeren gepresst, zentrifugiert (zwischen 3000 und 9000 rpm) im Labor für Wein- und Getränkeanalytik zwischen 30004000 rpm, filtriert (Spritzenfilter Minisart $5 \mu \mathrm{m}$, Sartorius) und mittels FTIR (FOSS ${ }^{\circledR}$ ) auf folgende Inhaltsstoffe analysiert: Mostgewicht, ${ }^{\circ} \mathrm{KMW}$, pH-Wert, Gesamtsäure, Apfelsäure, Weinsäure, HVS, Aminostickstoff, Ammoniumstickstoff.

Der Tag des Jahres an dem in den einzelnen Jahren 16 bzw. $18{ }^{\circ} \mathrm{KMW}$ erreicht wurden, wurde rechnerisch durch Interpolation der Daten ermittelt. Die Verrechnung der Daten und grafische Darstellung erfolgte mit IBM ${ }^{\circledR}$ SPSS Statistics sowie mit Microsoft Excel.

\section{ERGEBNISSE}

\section{ZUCKERGEHALTE}

Da die Entwicklungen der Zuckergehalte und auch der anderen Parameter in den einzelnen Anlagen weitestgehend ähnlich verlaufen, werden jeweils nur einige Beispiele dargestellt und näher besprochen. Die Abbildungen 1, 2 und 3 zeigen jeweils alle verfügbaren Einzelmessungen der betreffenden Lage auf. Die Sorte Weißburgunder in Eppan Berg (Abb. 1) wies in den Jahren vor der Jahrtausendwende (blaue Punkte) am 1. September (Tag 244 des Jahres, gekennzeichnet durch die rote Linie) Zuckergehalte zwischen 8 und $14^{\circ} \mathrm{KMW}$ auf. Nach der Jahrtausendwende (grüne und rote Punkte) lagen die Zuckerwerte zum 1. September bereits zwischen etwa 13 bis fast $19{ }^{\circ} \mathrm{KMW}$. Man erkennt in der Grafik sehr gut, wie die Zuckergehalte in der Erhebungszeit in den einzelnen Jahren etwas abweichen, es immer kühlere und wärmere Jahre gibt, sich z.B. grüne und blau markierte Jahre teilweise überschneiden, aber generell liegen die Jahre nach der Jahrtauendwende vermehrt im Bereich der höheren Zuckergehalte. Auch wurden in vielen Jahren der letzten beiden Jahrzehnte sehr hohe Zuckergehalte um die 19-20 ${ }^{\circ} \mathrm{KMW}$ erreicht und das meist bereits in der ersten Septemberhälfte, zwischen Tag 244 und 260 des Jahres. Vor der Jahrtausendwende war dies kaum der Fall.

Abbildung 1 zeigt zudem die Zuckergehalte aller Erhebungen des Standortes Terlan (Terlan Eyrl) auf. Es handelt sich hier um einen deutlich wärmeren Standort als Eppan Berg. Entsprechend findet hier die Reifeentwicklung überwiegend bereits vor dem 1. September statt. Die Zuckergehalte lagen am 1 . September in den Jahren vor der Jahrtausendwende zwischen etwa 13 und $16^{\circ} \mathrm{KMW}$ und danach zwischen 16 und $19^{\circ} \mathrm{KMW}$. Besonders vor der Jahrtausendwende wurden teilweise auch nur mäßige Zuckergradationen erzielt. In den letzten zwei Jahrzehnten lag der Erntetermin teilweise bereits um den 1 . September, bis Mitte September war aber auch in früheren Jahren meist der Lesetermin erreicht worden. Außerdem fällt noch auf, dass in besonders frühen Jahren, in denen die $18{ }^{\circ} \mathrm{KMW}$ bereits um den 230. Tag des Jahres, also etwa Mitte August vorlagen, der weitere Zuckerzuwachs auch in der Spalieranlage in den Jahren nach der Jahrtausendwende, dann eher flach verläuft. Die $20^{\circ} \mathrm{KMW}$, die in der deutlich kühleren Lage in Eppan Berg fast regelmäßig erzielt wurden, scheinen in der deutlich wärmeren Lage Terlan eher nicht zustande zu kommen.

Sehr stark verändert haben sich die Zuckergehalte auch bei der Sorte Vernatsch. Abbildung 1 zeigt alle verfügbaren Werte der Lage St. Magdalena, Bozen. Auch hier sind die Zuckerwerte zum 1. September heute deutlich höher als Mitte der 80er Jahre des letzten Jahrhunderts. Sie schwanken zwischen knapp $10{ }^{\circ} \mathrm{KMW}$ und gut $18{ }^{\circ} \mathrm{KMW}$. Die Ernte hat sich nur in einigen Jahren verfrüht, dafür wird aber in den letzten Jahren mit sehr hohen Zuckergehalten zwischen 18 und $20^{\circ} \mathrm{KMW}$ gelesen, was vor der Jahrtausendwende nur in Ausnahmefällen so war.

\section{GESAMTSÄURE}

Bei den Werten der Gesamtsäure zeigt sich ein etwas anderes Bild. Während die Werte vor dem 1. September in der Lage Eppan Berg (Abb. 2) noch weit auseinander lagen, näherten sie sich mit zunehmender Reife immer weiter an. Von wenigen Ausnahmen abgesehen, lagen die Werte in Nähe zur Ernte zwischen 5-10 g Gesamtsäure pro I Most. In einigen sehr warmen Jahren der letzten zwei Jahrzehnte fielen die Säurewerte zwar frühzeitig auf Werte um 5-6 g/l ab, in etlichen Fällen wurden aber auch in den letzten zwei
Jahrzehnten Säuregehalte erzielt, wie sie auch vor der Jahrtausendwende nicht selten vorkamen. So kann gesagt werden, dass sich in Summe die Säurewerte zur Lese oder in Nähe zur Lese nicht so deutlich verändert haben, wie dies bei den Zuckergehalten zu beobachten ist.

Ähnliches gilt für die in Abbildung 2 ebenfalls dargestellten Säuregehalte bei Weißburgunder in Terlan. Auch hier näherten sich die Gehalte in Nähe zur Lese deutlich aneinander an. Deutlicher als in der kühleren Lage Eppan Berg sind hier in den letzten zwei Jahrzehnten einige Jahre zu erkennen in denen die Gesamtsäuregehalte früh, noch im Monat August, auf Werte um 5-6 g/l abfielen. In den letzten zwei Jahrzehnten fehlen gegen Ende der Reifeentwicklung zwar Jahre mit höheren Säuregehalten um $10 \mathrm{~g}$, wie sie vor der Jahrtausendwende zum Teil vorkamen, aber in nicht wenigen Fällen wurden vor der Jahrtausendwende ähnliche Gesamtsäurewerte wie in den 80er und 90er Jahren des letzten Jahrhunderts erzielt. Auch hier gilt also, dass sich die Gesamtsäurewerte zur Lese im Untersuchungszeitraum nicht so eindeutig verändert haben wie die Zuckergehalte.

Ein leicht anderes Bild zeigt Abbildung 2 bei der Sorte Vernatsch. Hier fällt die Gesamtsäure schon früh in der Reifeentwicklung auf eher niedrige Werte ab und liegt zum 1. September bereits bei Werten zwischen 4$11 \mathrm{~g} / \mathrm{l}$. Auch hier finden sich Jahre nach der Jahrtausendwende mit ausnehmend niedrigen Gesamtsäuregehalten um $4 \mathrm{~g} / \mathrm{l}$ und einige mit überdurchschnittlich hohen Werten um $8 \mathrm{~g} / \mathrm{l}$ vor der Jahrtausendwende. Dazwischen mischen sich die Jahre aber kunterbunt durcheinander und es werden in der Nähe zur Lese in den letzten beiden Jahr zehnten ähnliche Gesamtsäurewerte erzielt, wie sie in den zwei vorhergehenden Jahrzehnten auch vorgekommen sind. Zwar werden diese Werte nun etwas früher erreicht als in den 80er Jahren des letzten Jahrhunderts, aber absolut gesehen liegen heute viele Werte auf ähnlichem Niveau wie früher.

\section{PH-WERT}

Beim $\mathrm{pH}$-Wert ergibt sich eine ähnliche Situation wie bei der Gesamtsäure. Zwar liegen die Werte zum 1. September in den Jahren nach der Jahrtausendwende deutlich höher als vorher, betrachtet man aber die Werte gegen Ende der Reifeperiode in Nähe zur Lese, so sind sie nur in einigen sehr warmen Jahren angestiegen. Sie lagen vor der 
Jahrtausendwende in einigen Jahren leicht tiefer als in späteren Jahren, in der Mehrzahl der Jahre lagen sie aber vor und nach der Jahrtausendwende auf ähnlichem Niveau (Abb. 3). Bei der Sorte Vernatsch in der sehr warmen Lage in St. Magdalena werden Werte bis knapp 3,8 erreicht, bei Weißburgunder in Eppan Berg liegen die pH-Werte immer unter 3,5 und in Terlan, in der für Weißburgunder sehr warmen Lage, steigen die höchsten Werte leicht über diesen Wert an, die überwiegende Mehrheit der Werte liegt aber unter 3,4. Am ehesten ist in der sehr warmen Lage Terlan ein Anstieg der pHWerte in der Nähe zum Lesetermin in den letzten zwei Jahrzehnten gegenüber den Jahren davor, zu erkennen. In allen Anlagen aber, in den hier dargestellten, wie auch in denen die hier nicht dargestellt sind, hebt sich im $\mathrm{pH}$-Wert ein Jahr besonders ab, nämlich das Jahr 2003. In den Zuckerwerten, wie auch bezüglich der zeitlich frühen Reifentwicklung fällt 2003 nicht so deutlich auf. Inzwischen wurden in mehreren Jahren ähnlich früh im Jahr, ähnlich hohe Zuckergehalte wie 2003 erreicht. Aber die pH-Werte des Jahres 2003 sind fast bei allen Sorten und in allen Standorten die höchsten welche je beobachtet wurden.

\section{DIE ZEITLICHE VERFRÜHUNG DER REIFEENTWICKLUNG}

Um diese darzustellen und die einzelnen Jahre miteinander vergleichen zu können, wurde der Termin errechnet an dem die Rebsorten, je nach Sorte und erreichter Reife, einen Zuckergehalt von 16 oder $18^{\circ} \mathrm{KMW}$ erreicht haben. Aus den Abbildungen 4 a-c kann abgelesen werden, um wieviele Tage sich die Zuckereinlagerung verfrüht hat. So wurde im Jahre 2019 bei den Sorten Sauvignon, Chardonnay und Ruländer der Zuckergehalt von 16 bzw. $18^{\circ} \mathrm{KMW}$ etwa 20 Tage früher erzielt als Mitte bis Ende der 80ziger Jahre des letzten Jahrhunderts, bei Gewürztraminer, der Weißburgunder-Anlage in Terlan und der Blauburgunder-Anlage in Kastelbell waren es etwa 25 Tage. Interessant ist aber, dass die Weißburgunder-Anlage in Eppan Berg und die Blauburgunder-Anlage in Mazzon eine wesentlich größere Verfrühung der Zuckereinlagerung um etwa 38-40 Tage aufweisen. Ebenfalls unterschiedlich ausgeprägt sind die Zuckerentwicklungen in den VernatschAnlagen. Die drei Anlagen Kaltern Barleit, Eppan Kreit und Marling Schickenburg weisen eine sehr ähnliche Entwicklung auf mit 35-37 Tagen Vorsprung in der Zuckereinlagerung, Kaltern See zeigt 40 Tage und die
Anlage in Bozen St. Magdalena 50 Tage frühere Entwicklung der Zuckereinlagerung auf

Das bedeutet, dass etwa Mitte der 80er Jahre des letzten Jahrhunderts bei Vernatsch in den drei höheren Lagen Kaltern Barleit, Eppan Kreit und Marling Schickenburg ein Zuckergehalt von $16{ }^{\circ} \mathrm{KMW}$ wenn überhaupt, dann durchschnittlich erst um den 15.-20. Oktober erreicht wurden. Heute ist dieser Zuckergehalt um den 10.-15. September zu erwarten. Bei Vernatsch in St. Magdalena wurden Mitte der 80er Jahre die $16^{\circ} \mathrm{KMW}$ in den ersten Oktobertagen erreicht, inzwischen ist es in den meisten Jahren schon im August so weit.

Bei der Sorte Weißburgunder wurden in Eppan Berg Mitte der 80er Jahre die Zuckergehalte von $16{ }^{\circ} \mathrm{KMW}$ durchschnittlich zwischen 25 . und 30 . September erreicht, inzwischen trifft dies schon um den 20. August zu. In der wärmeren Weißburgunder-Lage in Terlan waren die $16{ }^{\circ} \mathrm{KMW}$ früher um den 10. September gegeben, heute bereits um Mitte August.

\section{DAS VERHÄLTNIS VON ZUCKER ZU GESAMTSÄURE UND VON GE- SAMTSÄURE ZU PH-WERT}

Eine weitere Frage, die sich stellt, ist, ob sich das Verhältnis des Zuckergehaltes zur Gesamtsäure oder der Gesamtsäure zum pHWert mit zunehmender Klimaerwärmung verändert hat. Natürlich ist es so, dass im Falle einer Lese mit höherem Zuckergehalt auch eine niedrigere Gesamtsäure zu erwarten ist. Aber wie lagen bei gleichen Zuckerwerten die Säuregehalte früher und wie sind sie heute? Die Abbildungen 5 a-c zeigen diese Zusammenhänge bei den Sorten Weiß- und Blauburgunder, Ruländer, Gewürztraminer, Chardonnay, Weißem Sauvignon und Vernatsch. In den meisten Fällen wurden die Werte vor dem Jahre 2000 mit jenen nach dem Jahre 2000 verglichen, be Ruländer wurde bis 2004 mit ab 2005 in Beziehung gebracht, da mit 2005 ein Wechsel der Anlage stattgefunden hat.

Erstaunlicher Weise zeigen die Ergebnisse einen einheitlichen Trend auf: Bei gleichen Zuckerwerten wurden nach dem Jahr 2000 immer eher höhere Gesamtsäurewerte gefunden als vorher und der $\mathrm{pH}$-Wert ist bei gleicher Gesamtsäure nach dem Jahr 2000 eher niedriger als vorher. Dies ist zumindest im kritischen Bereich so, also bei hohen Zuckergehalten ist heute eher mehr Gesamtsäure zu finden und bei niedrigen $\mathrm{Ge}$ - samtsäuregehalten ist der $\mathrm{pH}$-Wert eher niedriger als vor dem Jahr 2000 (Abb. 6 a-c)

So zeigt die Sorte Chardonnay bei einem Zuckergehalt von $17{ }^{\circ} \mathrm{KMW}$ nach dem Jahr 2000 etwa durchschnittlich 1,5 g höhere Gesamtsäuregehalte als in den Jahren davor, bei den Sorten Vernatsch und Weißburgun der sind es ca. 2 g höhere Gesamtsäuregehalte in den letzten beiden Jahrzehnten gegenüber früher. Bei einigen Sorten ist kein praktisch relevanter Unterschied zwischen vor und nach dem Jahr 2000 zu sehen, aber wie bereits erwähnt ist die Tendenz bei allen Sorten dieselbe.

Besonders ausgeprägt ist diese Veränderung bei der Sorte Weißburgunder in Eppan Berg (nicht abgebildet). Hier wurde bis zum Jahre 2004 eine eher ertragreiche Perglanlage beprobt und ab 2005 eine Spalieranlage mit niedrigerem Ertragsniveau. Hier sind in den letzten zwei Jahrzehnten bei einem Zuckergehalt von $18{ }^{\circ} \mathrm{KMW}$ etwa $2 \mathrm{~g} / \mathrm{l}$ höhere Gesamtsäurewerte und bei $7 \mathrm{~g} / \mathrm{l}$ Gesamtsäure ein um etwa den Wert 0,2 niedrigerer $\mathrm{pH}$ Wert festzustellen.

\section{DISKUSSION}

Es ist augenscheinlich, dass heute die Reife früher einsetzt und früher geerntet wird als vor drei Jahrzehnten. Dass es sich dabei aber um eine Verfrühung der Zuckereinlagerung von drei Wochen handelt gegenüber den 80er Jahren des letzten Jahrhunderts, überrascht einerseits, andererseits werden von den allermeisten Weinbaugebieten Europas ähnliche große Verschiebungen aufgezeigt. In einigen hier vorliegenden Fällen ist die Verfrühung noch deutlich größer, bei der Sorte Vernatsch in St. Magdalena beträgt sie 50 Tage. Aus den vorhandenen Daten kann nicht systematisch rekonstruiert werden, worauf diese Unterschiede bei den einzelnen Lagen zurückzuführen sind. Bei der Anlage St. Magdalena fällt allerdings auf, dass sie in den letzten Jahren ein sehr niedriges Ertragsniveau aufwies. Nicht ganz so ausgeprägt, aber in der Tendenz ähnlich, verhält es sich auch beim Weißburgunder Eppan Berg, die heutige Anlage liegt im Ertragsniveau deutlich niedriger als die ursprünglich beprobte Perglanlage. Es ist daher anzunehmen, dass die heutigen niedrigeren Erträge teilweise zur festgestellten Reifeverfrühung mit beitragen. Eine erste deutliche Reduzierung der Erträge wurde Ende der 80er und in den 90er Jahren durchgeführt, aber bis herauf in die heutige Zeit wurden, für die Erzeugung besonders hochwertiger Weine, wei- 
tere Ertragsreduzierungen umgesetzt. Weitere zugrunde liegende Ursachen zeichnen sich nicht $a b$, es sind z. B. nicht immer die kühleren Lagen, welche eine größere oder kleinere Reifeverfrühung aufweisen. Zu erwarten gewesen wäre eine etwas geringere Verfrühung in den bereits klimatisch sehr günstigen Lagen der jeweiligen Sorte. Bei Vernatsch war die mit Abstand größte Reifeverfrühung aber in der sehr warmen Lage in Bozen gegeben, bei Weißburgunder liegt eine größere Reifeverfrühung in der höheren und kühleren Lage in Eppan Berg vor.

In allen Fällen liegt aber eine frühere Entwicklung der Zuckereinlagerung von etwa 20-25 Tagen vor, in einer Anlage beträgt diese nur 19 Tage, in den meisten Fällen etwas mehr. In einem Fall beträgt die Reifeverfrühung sogar 50 Tage. Das bedeutet nun nicht, dass inzwischen auch durchschnittlich entsprechend früher geerntet wird, denn in den allermeisten Fällen werden die Trauben heute mit einem deutlich höheren Zuckergehalt als früher gelesen.

Besonders erstaunlich erscheinen die Entwicklungen der Zucker-Säure- und der Säure-pH-Wert-Relation. An und für sich wäre heute bei gleichem Zuckergehalt eine niedrigere Gesamtsäure zu erwarten gewesen. Die Trauben reifen heute früher, also bei wärmeren Tages- und Nachttemperaturen, wodurch eine niedrigere Äpfelsäure zu erwarten wäre [16], da sie sich bei höheren Temperaturen während der Reife schneller abbaut. Die hier vorliegenden Daten zeigen dies aber nicht, im Gegenteil, entweder liegen die Werte heute bei gleichem Zuckergehalt ähnlich wie früher oder die Säurewerte sind bei gleichen Zuckergehalten mehr oder weniger ausgeprägt höher als früher. Es gibt keinen einzigen Fall wo es umgekehrt ist und wo die Gesamtsäuregehalte bei gleichem Zuckergehalt heute im Durchschnitt der Jahre niedriger sind als früher. Zum Teil ist es auch so, dass bei gleichen Gesamtsäurewerten der $\mathrm{pH}$-Wert niedriger ist als vor Jahrzehnten. Dabei macht es keinen Unterschied, ob von Pergl- auf Spaliererziehung gewechselt wurde oder ob das Erziehungssystem weiterhin die Pergl geblieben ist. Am Erziehungssystem liegt es also nicht. Allein an der Ertragsreduzierung dürfte es auch kaum liegen, werden doch die heutigen niedrigen Erträge oft für die niedrigen Gesamtsäuregehalte mit verantwortlich gemacht. Bei den Ausdünnversuchen am Versuchszentrum Laimburg in den 80er Jahren des letzten Jahrhunderts gingen die niedrigeren Erträge tatsächlich mit eher niedrigeren Gesamtsäurewerten einher.
Warum wir, wie die vorliegenden Daten deutlich zeigen, heute eher höhere Gesamtsäurewerte und niedrigere $\mathrm{pH}$-Werte bei gleichen Zuckergehalten finden als früher, dürfte daher auf das Zusammenwirken der stattgefundenen Veränderungen zurückzuführen sein. Im Detail dürften dabei aber die heutige mäßigere Wuchsintensität der Anlagen, die vielfach ganz ausgesetzten Stickstoffdüngungen und die im Vergleich zu den 80er Jahren sehr begrenzte Ausbringung von Kalium eine Rolle spielen. So waren damals jährliche Gaben von bis zu $400 \mathrm{~kg} \mathrm{~K}_{2} \mathrm{O}$ pro Hektar, ausgebracht zu zwei Terminen, im Frühjahr und etwa zu Farbumschlag (Kalium für die Zuckereinlagerung!) keine Seltenheit. Entsprechende Mengen werden heute in Südtirol vielfach im Zeitraum von 10 oder noch mehr Jahren ausgebracht. Überhöhte Kaliumaufnahme führt bekanntlich zu höherem Ausfall von Weinsäure und zu höherem pH-Wert [17] [18]. Auch gibt es Berichte wonach eine höhere Stickstoffaufnahme den $\mathrm{pH}$-Wert der Moste und Weine erhöht [19].

Ein weiterer Faktor in diesem Zusammenhang könnte die heute grundlegend andere Situation bei den Temperatur- und Bodenwassergehalten sein. Die Niederschläge haben sich im Durchschnitt der Jahre in Südtirol kaum verändert. Allein aber durch die höheren Temperaturen und durch die heute oft fast vollkommen schneefreien Winter dürfte der Wasserverbrauch der Pflanzen und die stattfindende Verdunstung heute wesentlich größer als früher sein [5]. Es ist daher anzunehmen, dass inzwischen über weite Strecken der Vegetationsperiode und auch in der Reifephase der Trauben in den meisten Jahren größere Wasserdefizite vorliegen, als das in der Vergangenheit der Fall war. Auch dies dürfte sich auf die Kaliumund auch auf die Stickstoffaufnahme auswirken und damit auch die Gesamtsäure und den pH-Wert mit beeinflussen. Bei beiden Elementen, bei Kalium wie bei Stickstoff, wird die Aufnahme mit zunehmender Bodenaustrocknung erschwert.

Allerdings wird in Südtirol heute vielfach auch bewässert, während zu Beginn der hier aufgezeigten Erhebungen die Mehrzahl der Rebanlagen noch nicht mit entsprechender Technik ausgestattet waren. Bei der Bewässerung handelt es sich im Weinbau aber fast ausschließlich um Tröpfchenbewässerung, somit werden punktuelle Wassergaben verabreicht, welche einen begrenzten Feuchtkegel im Boden erzeugen. Es wird nicht der gesamte Wurzelraum der Reben durch- feuchtet und die obersten $10 \mathrm{~cm}$ Boden, also der Bereich mit dem höchsten Humusgehalt, wird kaum angefeuchtet. Somit wird auch in der Trockenphase kaum Stickstoff aus der Humusschicht der dauerbegrünten Weinbauböden Südtirols freigesetzt. Auch für die Kaliumaufnahme ist eine Tröpfchenbewässerung vermutlich in den meisten Fällen nicht mit einer Ganzbodenbefeuchtung wie sie bei natürlichen Niederschlägen erfolgt, zu vergleichen.

Eine Ausnahmeerscheinung unter den bisherigen heißen und trockenen Jahren stellt nach wie vor das Jahr 2003 dar. Obwohl inzwischen mehrere Jahre mit vergleichbar hohen und noch höheren Temperaturen währen der Vegetationsperiode und entsprechender Reifeverfrühung zu beobachten waren, sind die 2003 erzielten hohen pH-Werte der Moste immer noch unübertroffen. Die Zucker- und Gesamtsäurewerte heben sich hingegen nicht gleich deutlich ab. Wie es dazu kommt, bleibt noch eingehender zu untersuchen. Eventuell spielt dabei die ausnehmend lange Hitze- und Trockenphase im Jahre 2003, welche mehr oder weniger bis zur Ernte andauerte, eine Rolle. In den meisten Jahren treten in Südtirol, früher wie heute, vor der Ernte zumindest einzelne intensive Niederschlagsereignisse auf.

Laut dem Südtiroler Landesmeteorologen Dieter Peterlin war 2019 das wärmste Jahr in Bozen seit Beginn der Wetteraufzeichnungen im Jahre 1850. An 2. Stelle kommt das Jahr 2018, gefolgt von 2014 als das drittwärmste Jahr [20]. Weinbaulich gesehen war 2019 kein besonders frühes Jahr und vor allem 2014 war ein ausnehmend spätes Weinbaujahr. Höhere Jahres-Durchschnittstemperaturen bedingen somit nicht unbedingt auch eine frühere Traubenernte, vielmehr kommt es auf die Wettersituation zu den kritischen Phasen der Entwicklung der Rebe, vor und zur Blüte und während der Reifeperiode an.

Ein weiteres Phänomen scheint sich abzuzeichnen. Wie die Abbildungen 4 a-c erkennen lassen, scheinen die Jahrgangsschwankungen in den letzten ein bis zwei Jahrzehnten eher zuzunehmen. Jahre mit ausnehmend früher Reife wechseln mit eher späteren Jahren ab. Klimaforscher nehmen an, dass dies eine Auswirkung des Klimawandels ist und in Zukunft vermehrt Wechsel zwischen kühleren und warmen Phasen zu verzeichnen sein könnten. Es erscheint sogar möglich, dass es mit fortschreitender Klimaänderung, wieder Jahre oder auch mehrere 
Jahre in Folge mit deutlich kühleren Bedingungen geben könnte [21].

Wie die dargestellten Ergebnisse zeigen, haben in den vergangenen dreieinhalb Jahrzehnten beachtliche Veränderungen in der Reifeentwicklung der Trauben stattgefunden. Durchschnittlich ernten wir heute deutlich früher im Jahr Trauben mit höheren Zucker- und niedrigeren Gesamtsäuregehalten als früher. Das Zucker- Säureverhältnis der Trauben hat sich bisher, bei gleichem Zuckergehalt und im Durchschnitt der Jahre, aber nicht verschlechtert. Die bisherigen Veränderungen der Reifeentwicklung, soweit es die hier vorliegenden Daten erkennen lassen, können daher als groß und einschneidend bezeichnet werden, nicht aber als ausgesprochen negativ. Selbst in den sehr warmen Lagen Südtirols können bisher, trotz der beachtlichen Reifeverfrühung, immer noch Trauben mit geeigneten Voraussetzungen für die Weinbereitung erzeugt werden. Es stellt sich allerdings die Frage, wie lange dies so bleiben wird. Zunehmend Probleme bereiten könnten auch die Extremwetterlagen. Einzelne Jahre mit extremem Witterungsverlauf haben sich bereits, wie aufgezeigt, stark vom durchschnittlichen Verlauf der Reife abgehoben. Man denke an die auffallend hohen $\mathrm{pH}$ Werte im anhaltend heißen und trockenen Jahr 2003 oder die unterdurchschnittliche und späte Reifeentwicklung im warmen, aber feuchten Jahr 2014. Die prognostizierte, weitere Zunahme extremer Wetterereignisse beinhaltet für den Weinbau der Zukunft daher jedenfalls ein erhöhtes Risiko.

\section{ZUSAMMENFASSUNG}

Seit dem Jahr 1985 werden am Versuchszentrum Laimburg alljährlich im Herbst, in den Wochen vor der Reife, bei den wichtigsten Südtiroler Rebsorten Tests durchgeführt, um die Reifesituation zu ermitteln und den Weinbaubetrieben die Planung der Ernte zu erleichtern. Die dabei gewonnenen Daten stellen zugleich eine interessante Dokumentation der Veränderungen in diesem Zeitraum dar. Neben der Klimaänderung mit einer Zunahme der durchschnittlichen Temperaturen waren die letzten dreieinhalb Jahrzehnte in Südtirol auch von großen Veränderungen im Weinbau und insbesondere durch das Streben nach höherwertiger Trauben- und Weinqualität geprägt. Derselbe Zuckergehalt der Trauben wird heute um 19-50 Tage früher erreicht als zu Beginn der Reifetests im Jahre 1985. In den allermeisten Fällen ist eine Reifeverfrühung von etwa 3 Wochen zu erkennen. Aus den vorliegenden Daten kann nicht ermittelt werden wieweit diese Veränderungen auf die veränderten Klimabedingungen oder auf Veränderungen im Anbau zurückzuführen sind. Letztere dürften auch eine Rolle spielen, insbesondere in den Fällen mit überdurchschnittlicher Reifeverfrühung. Die Gesamtsäurewerte der Moste sind bei gleichem Zuckergehalt nicht abgefallen bzw. auch der $\mathrm{pH}$-Wert ist bei gleichem Zuckergehalt nicht angestiegen. Dies wäre eigentlich, aufgrund der inzwischen wärmeren Bedingungen während der Traubenreife, zu erwarten gewesen. Vielmehr ist es so, dass bei gleichem Zuckergehalt die Gesamtsäurewerte entweder ähnlich geblieben sind oder heute höher liegen als früher. Teilweise ist be gleichen Gesamtsäurewerten auch der pH-Wert heute niedriger als früher.

\section{RIASSUNTO}

Dal 1985 al Centro di Sperimentazione Laimburg, nelle settimane prima della vendemmia, vengono eseguiti test di maturazione delle varietà di vite più importanti dell'Alto Adige. Lo scopo è di monitorare lo stato di maturazione e facilitare alle aziende viticole la programmazione della vendemmia. I dati risultanti rappresentano inoltre una documentazione interessante dei cambiamenti avvenuti in questo arco di tempo. Oltre al cambiamento climatico con un aumento delle temperature medie, gli ultimi tre decenni in Alto Adige sono stati caratterizzati da un crescente orientamento verso la produzione viticola di alta qualità. Oggi, rispetto all'anno 1985, lo stesso contenuto di zuccheri dell'uva viene raggiunto da 19-50 giorni prima. Nella gran parte de casi si può notare un anticipo della maturazione di circa tre settimane. Dai dati disponibili non è possibile stabilire in quale misura questo anticipo è dovuto al cambiamento climatico e quanto contribuiscono tali cambiamenti alla coltivazione de vigneti. È però probabile che anche questi ultimi abbiano un certo ruolo, soprattutto nei casi con un anticipo della maturazione sopra la media. A parità di grado zuccherino, i valori di acidità totale dei mosti non sono calati, così come i valori di pH non sono aumentati. A causa di una maturazione anticipata in condizioni climatiche più calde, ci si sarebbe aspettati uno scenario diverso: i dati dimostrano invece che a parità di grado zuccherino i valori di acidità totale sono invariati o addirittura aumentati rispetto al passato. In alcuni casi, a pari acidità totale, i valori di pH dei mosti sono minori rispetto al passato. 


\section{LITERATUR}

[1] Schultz H.R. (2000). Climate change and viticulture: A European perspective on climatology, carbon dioxide and UV-B effects. Australian Journal of Grape and Wine Research 6 (1), 2-12, DOI: 10.1111/j.17550238.2000.tb00156.x.

[2] Mira de Orduna R. (2010). Climate change associated effects on wine quality and production. Food Research International 43 (7), 1844-1855, DOI: 10.1016/j.foodres.2010.05.001.

[3] Jones G.B., White M.A., Cooper O.R. et al (2005). Climate change and global wine quality. Climatic Change 73, 319-343, DOI: 10.1007/s10584-005-4704-2.

[4] Petrie P., Sadras V. (2008). Advancement of grapevine maturity in Australia between 1993 and 2006: Putativecauses, magnitude of trends and viticultural consequences. Australian Journal of Grape and Wine Research 14, 33-45, DOI: 10.1111/j.17550238.2008.00005.x.

[5] Van Leeuwen C., Darriet P. (2016). The impact of climate change on viticulture and wine quality. Journal of Wine Economics 11 (1) 150-167, DOI: 10.1017/jwe.2015.21.

[6] Van Leeuwen C., Trégoat O., Choné X. (2009) Vine water status is a key factor in grape ripening and vintage quality for red Bordeaux wine. How can it be assessed for vineyard management purposes? Journal international des sciences de la vigne et du vin 43 (1), 121-134, DOI: 10.20870/oenoone.2009.43.3.798.
[7] Duchêne E., Schneider C. (2005). Grapevine and climatic change. A glance at the situation in Alsace. Agronomy for Sustainable Development 25 (1), 93-99.

[8] Fraga H. (2019). Viticulture and Winemaking under Climate Change. Agronomy 9 (12) 783, DOI: 10.3390/agronomy9120783.

[9] Pons A., Allamy L., Schüttler A. et al. (2017). What is the expected impact of climate change on wine aroma compounds and their precursors in grape? OENO One 51 (23), 141-146, DOI: 10.3390/agronomy9120783.

[10] Peterlin D. (2018). Temperaturabweichungen Bozen 1850-2018. Retrieved September 10, 2020, from https://twitter.com/dieterpeterlin/status/1078600433395003392.

[11] Aichner M., Drahorad W., Menke F. et al. (2004). Gezielte Düngung im Obst- und Weinbau. Boden und Pflanzenernährung im Obstbau, Weinbau und Bioanbau. Land- und Forstwirtschaftliches Versuchszentrum Laimburg, Pfatten, Italien, hier p. 118.

[12] Raifer B. (2000). Essigfäule bei der Sorte Blauburgunder. Der Südtiroler Landwirt 54 (13), 42-43.

[13] Raifer B., Terleth J. (1997). Höhere Qualität durch Entblättern der Trauben. Obstbau Weinbau 34 (9), 240-241.

[14] Raifer B., Terleth J., Pedri U. (2004). Lb 36 und $\mathrm{Lb}$ 50, zwei neue Laimburger Klone der Sorte Weißer Sauvignon. Obstbau Weinbau 41 (2), 48-50.
[15] Raifer B., Terleth J., Pedri U. (2002). Blauburgunderklone. Obstbau Weinbau 39 (9), 235238.

[16] Keller M. (2015). The Science of Grapevines. Academic Press, Elsevier, London, UK, hier p. 249.

[17] Boulton R. (1980). The General Relationship Between Potassium, Sodium and $\mathrm{pH}$ in Grape Juice and Wine. American Journal of Enology and Viticulture 31 (2), 182-186.

[18] Ryser J.P., Aerny J., Murisier F. (1989). Fumure potassique de la vigne e aciditè du vin. Revue suisse de Viticulture, Arboriculture, Horticulture 21 (5), 319-323.

[19] Spayd S.E., Wample R.L., Evans R.G. et al. (1994). Nitrogen Fertilization of White Riesling Grapes in Washington. Must and Wine Composition. American Journal of Enology and Viticulture 45, 34-42.

[20] Athesia Druck (ed) (2019). 2019 war zweitwärmstes Jahr seit Messbeginn. Retrieved September 10, 2020, from https://www.stol.it/artikel/chronik/2019war-zweitwaermstes-jahr-seit-messbeginn.

[21] Sgubin G., Swingedouw D., García de Cortázar-Atauri I. et al. (2019). The Impact of Possible Decadal-Scale Cold Waves on Viticulture over Europe in a Context of Global Warming. Agronomy 9 (7) 397, DOI: 10.3390/agronomy9070397. 


\section{ANHANG: ABBILDUNGEN}

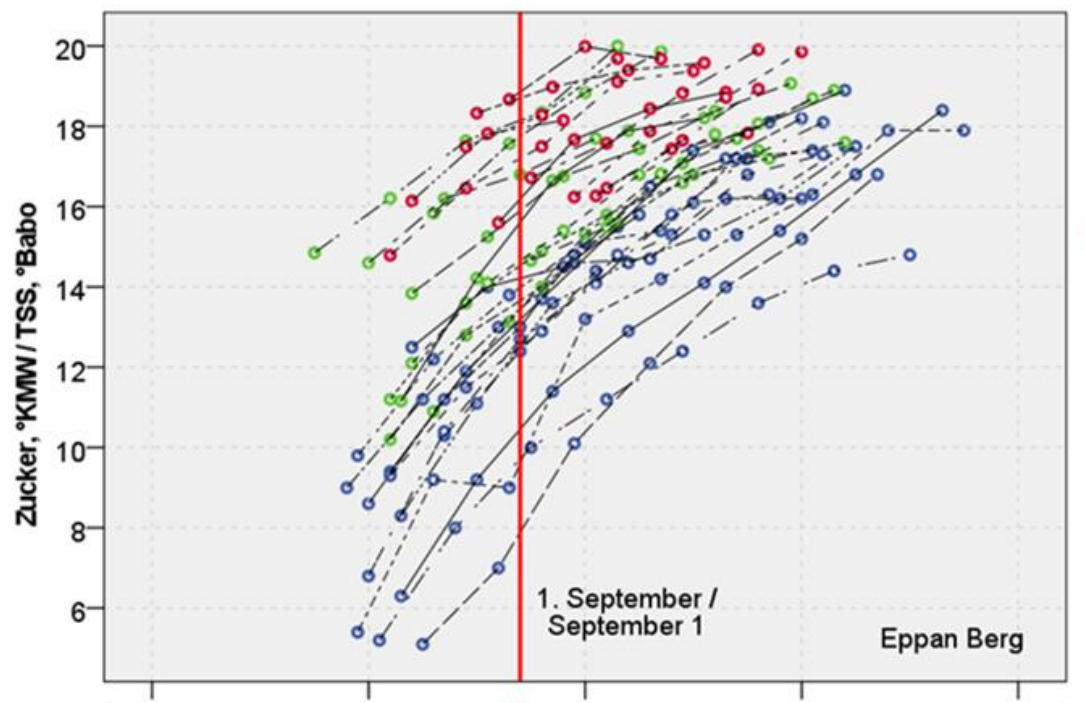
Jahr / year
- $1987-1999$
- 2000-2009
ㅇ 2010-2019

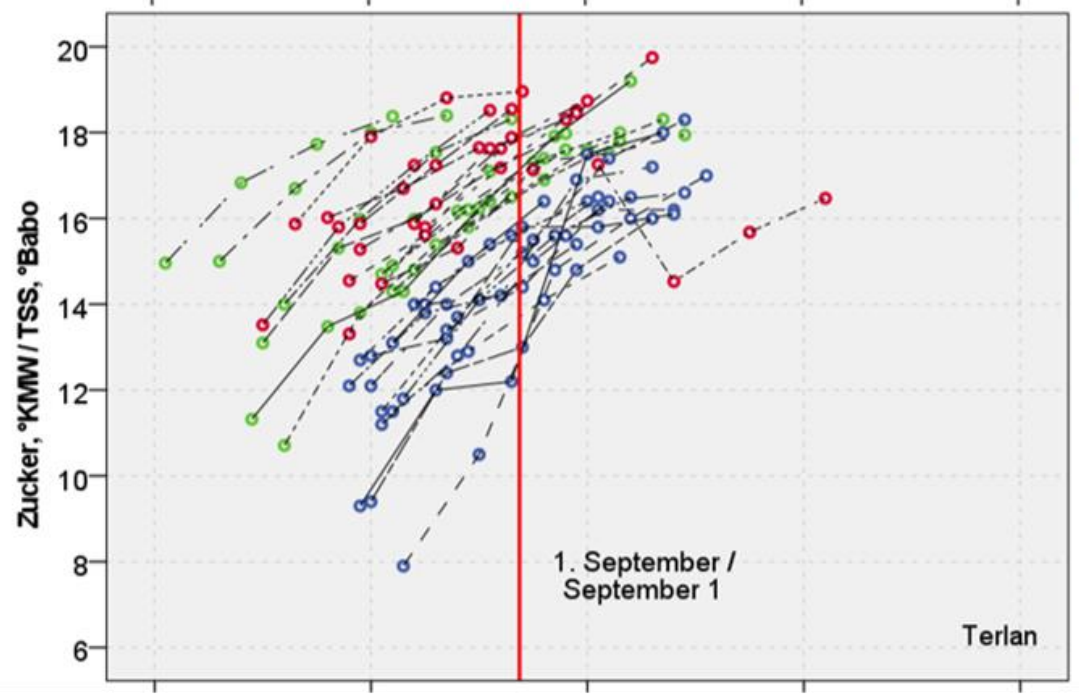

Jahr / year

ㅇ 1985-1999

○ 2000-2009

- 2010-2019

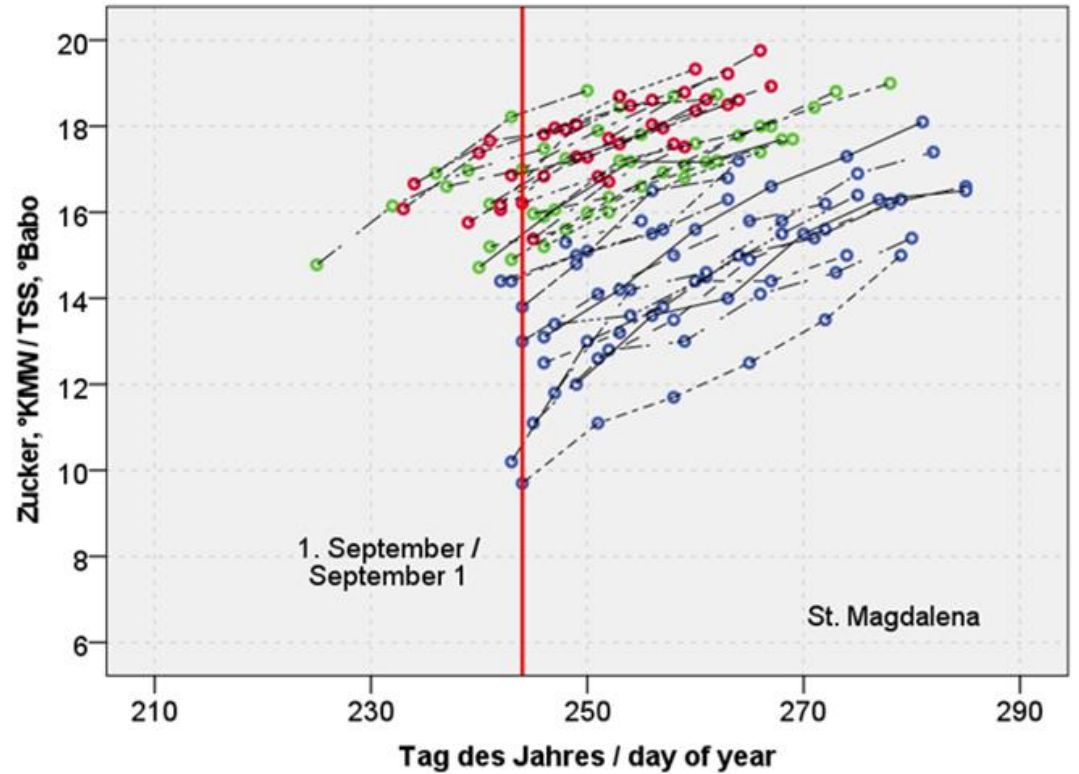

Jahr/year

- $1986-1999$

- $2000-2009$

- 2010-2019

Abb. 1: Alle verfügbaren Werte der Most-Zuckergehalte der Sorte Weißburgunder in den Erhebungsjahren in einer höheren und einer tiefen Lage (Eppan Berg und Terlan) und der Sorte Vernatsch in Bozen, St. Magdalena. // All available values of the must soluble solids in the survey years in a high and a low site of the variety Pinot blanc (Eppan Berg and Terlan) and of a Vernatsch vineyard in Bozen St. Magdalena. 

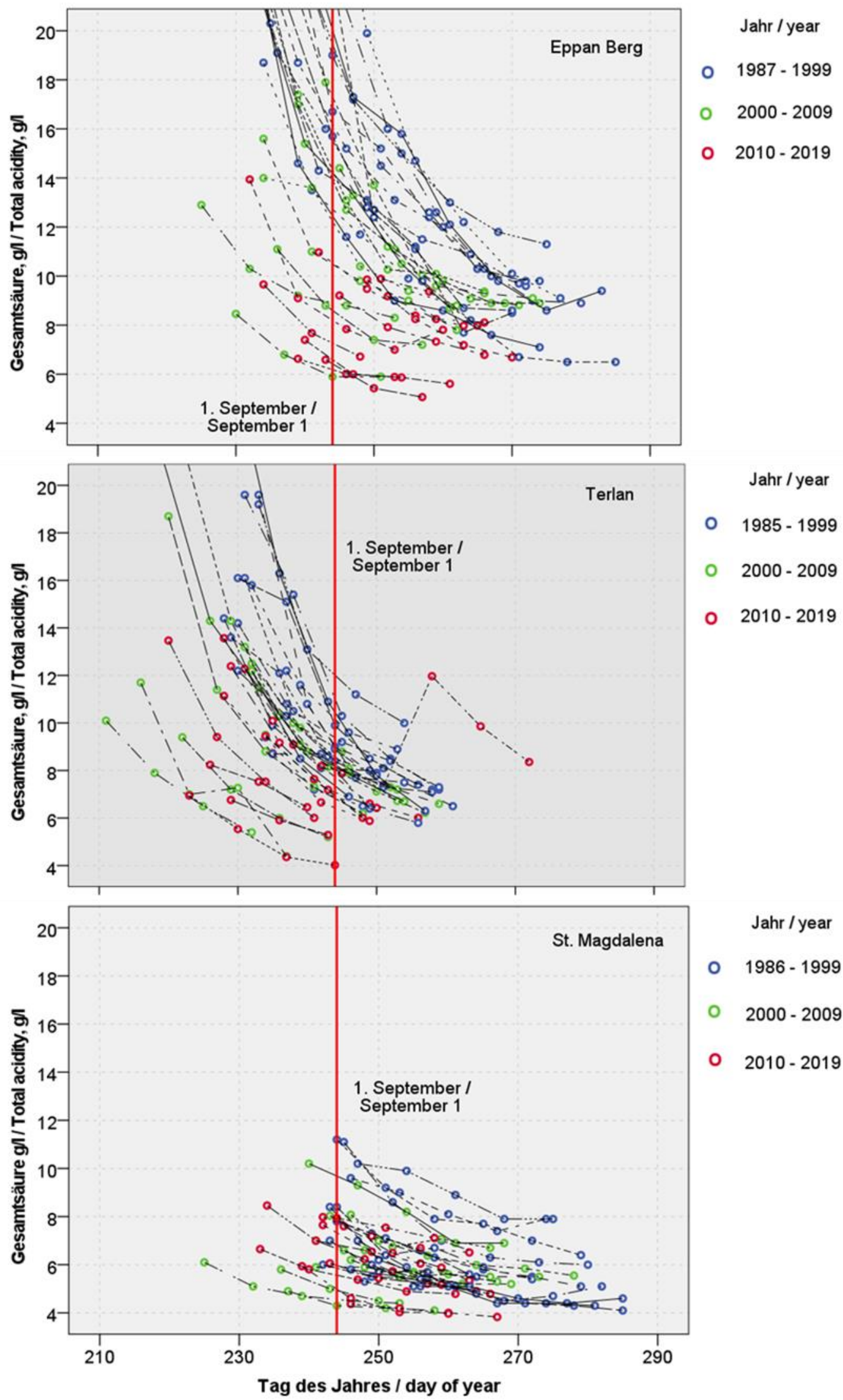

Abb. 2: Aller verfügbaren Werte der Most-Gesamtsäure in den Erhebungsjahren der Sorte Weißburgunder in einer höheren und einer tiefen Lage (Eppan Berg und Terlan) und der Sorte Vernatsch in Bozen, St. Magdalena. // All available values of must total acidity in the survey years in a high and a low site of the variety Pinot blanc (Eppan Berg and Terlan) and of a Vernatsch vineyard in Bozen St. Magdalena. 


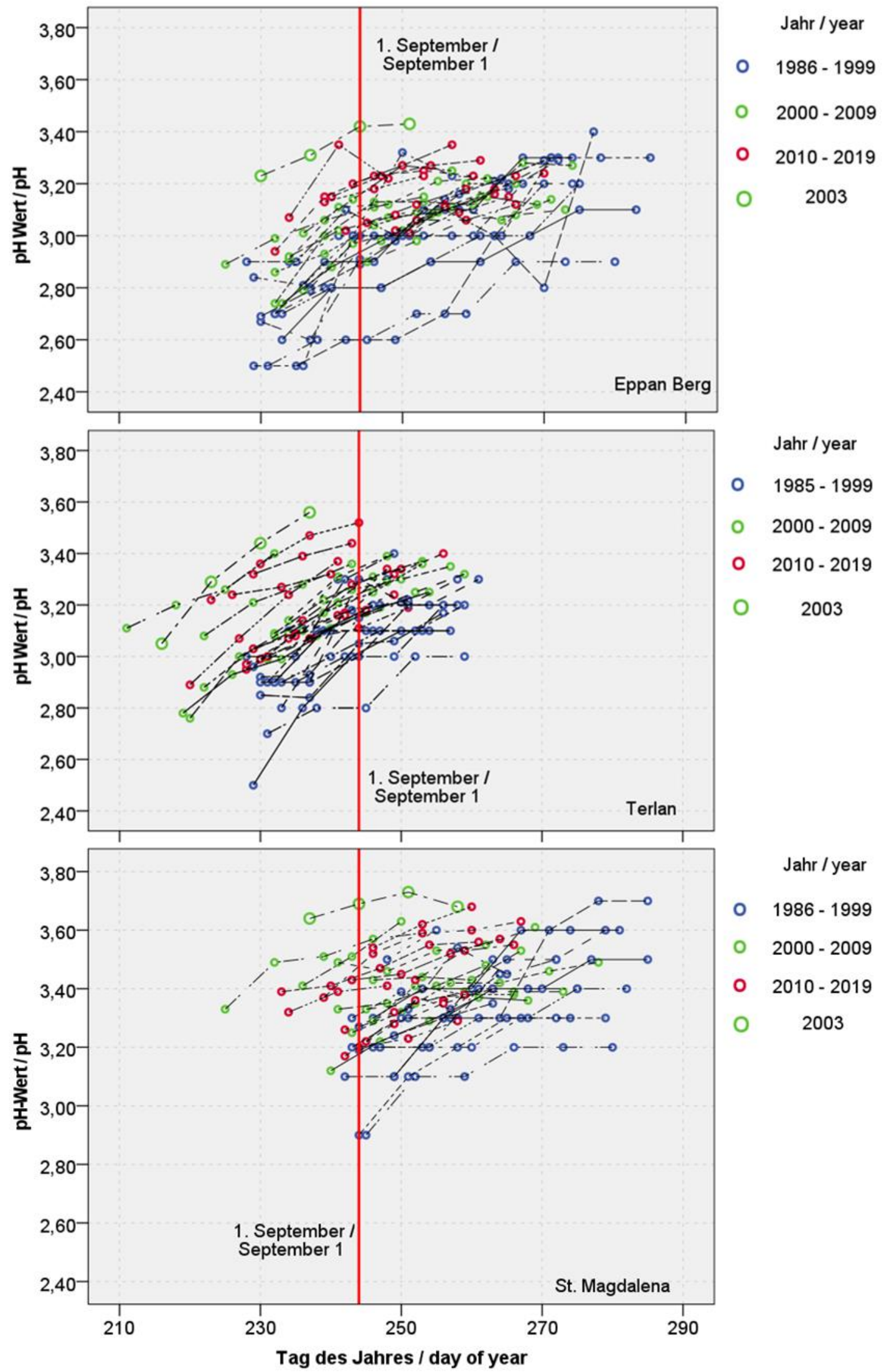

Abb. 3: Alle verfügbaren Werte der Most pH-Werte in den Erhebungsjahren der Sorte Weißburgunder in einer höheren und einer tiefen Lage (Eppan Berg und Terlan) und der Sorte Vernatsch in Bozen, St. Magdalena. // All available values of the must pH in the survey years in a high and a low site of the variety Pinot blanc (Eppan Berg and Terlan) and of a Vernatsch vineyard in Bozen St. Magdalena. 
Abb. 4 a-c: Tag des Jahres an dem in den einzelnen Jahren und Anlagen, je nach Sorte, ein Zuckergehalt von 16 oder $18^{\circ}$ Babo (KMW) erreicht worden ist. // Day of the year when in the single years and vineyards, according to the variety, TSS of 16 or $18{ }^{\circ}$ Babo have been riched.
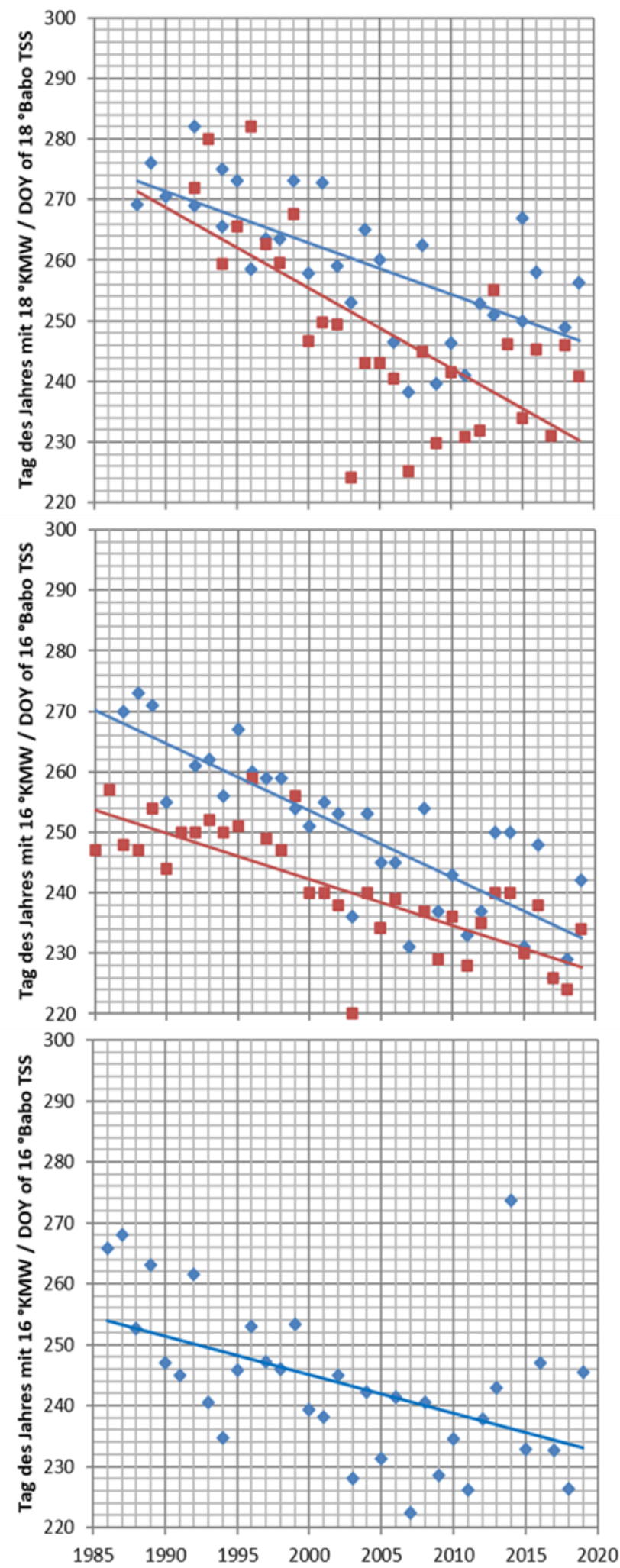

Abb. 4a.

\section{Blauburgunder}

- Kastelbe.

- Mazzon

— Linear (Kastelbe.)

— Linear (Mazzon)

\section{Weißburgunder}

- Berg Epp.

- Terlan

— Linear (Berg Epp.)

— Linear (Terlan)

\section{Chardonnay}

- Kurtinig

— Linear (Kurtinig) 


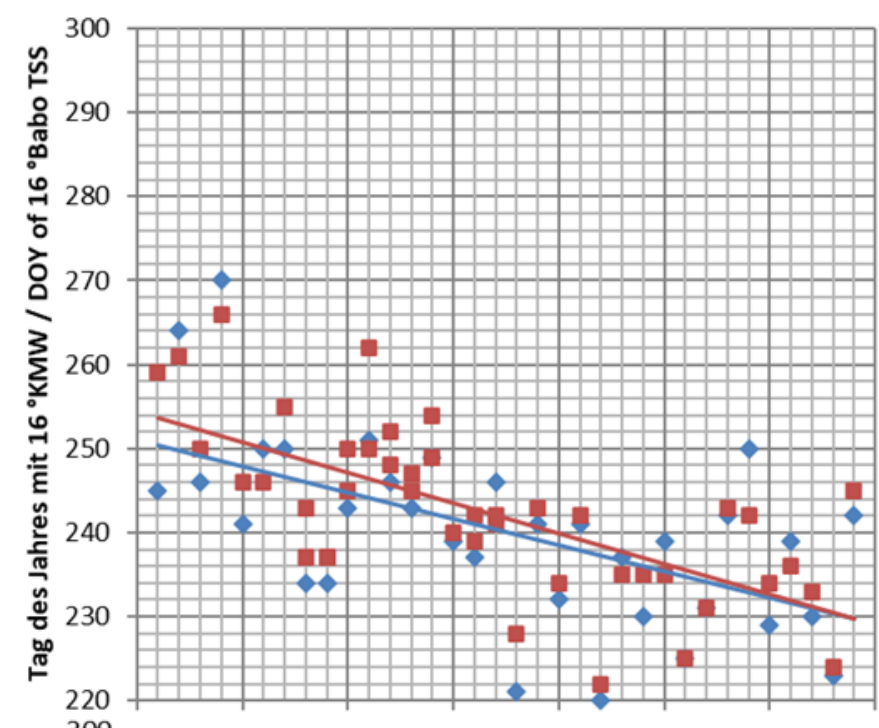

Ruländer
- Kurtinig
- Salurn
— Linear (Kurtinig)
— Linear (Salurn )

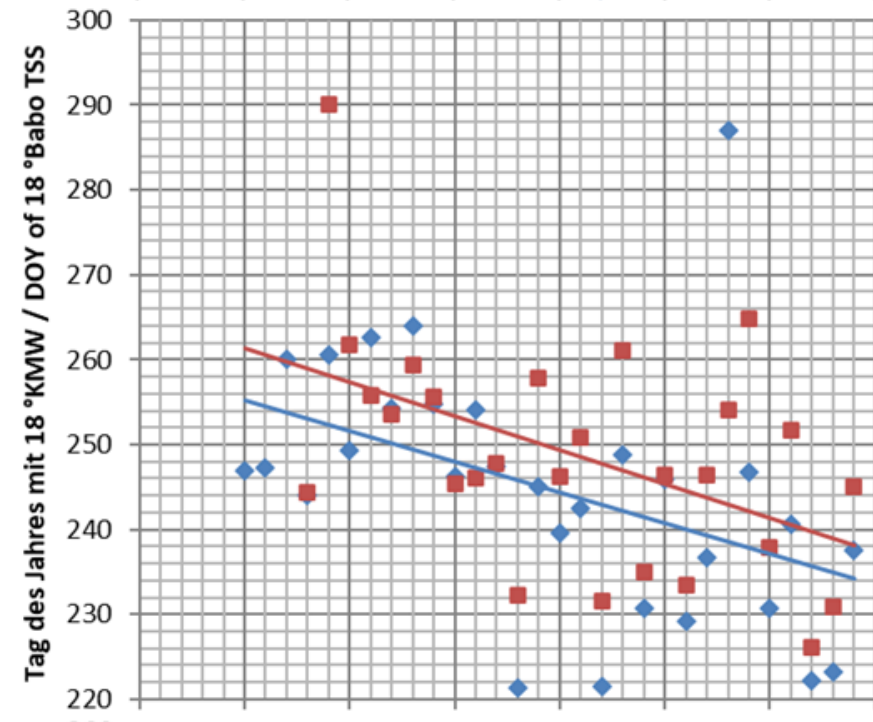

\section{Sauvignon}

- Terlan

- Girlan N.

— Linear (Terlan )

— Linear (Girlan N.)

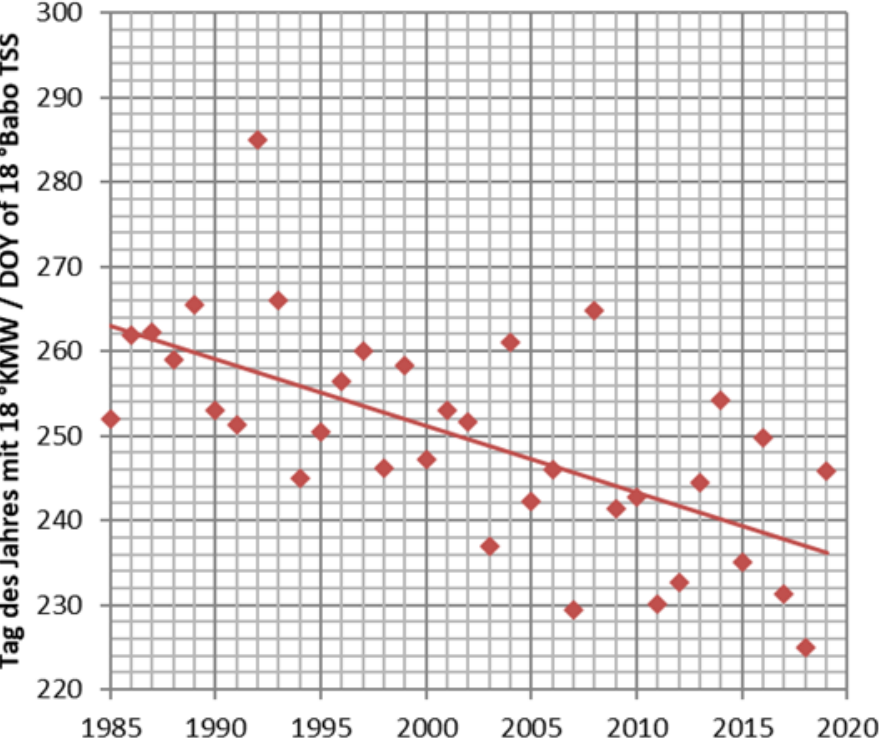

\section{Gewürztraminer}

- Tramin R.

— Linear (Tramin R.)

Abb. 4b. 


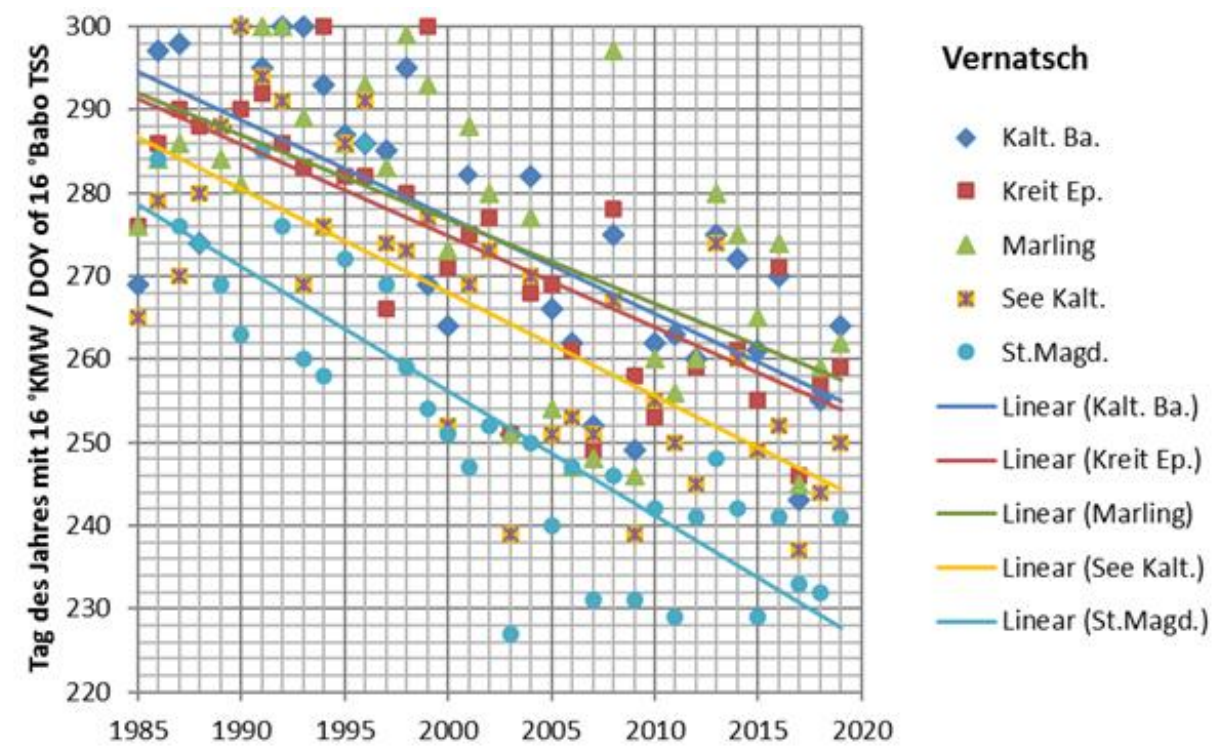

Abb. 4c. 
Abb. 5 a-c: Relationen der Most-Zuckergehalte zu den Gesamtsäurewerten der einzelnen Sorten und Anlagen. // Relations between must total soluble solids (TSS) and total acidity of the single varieties and sites.
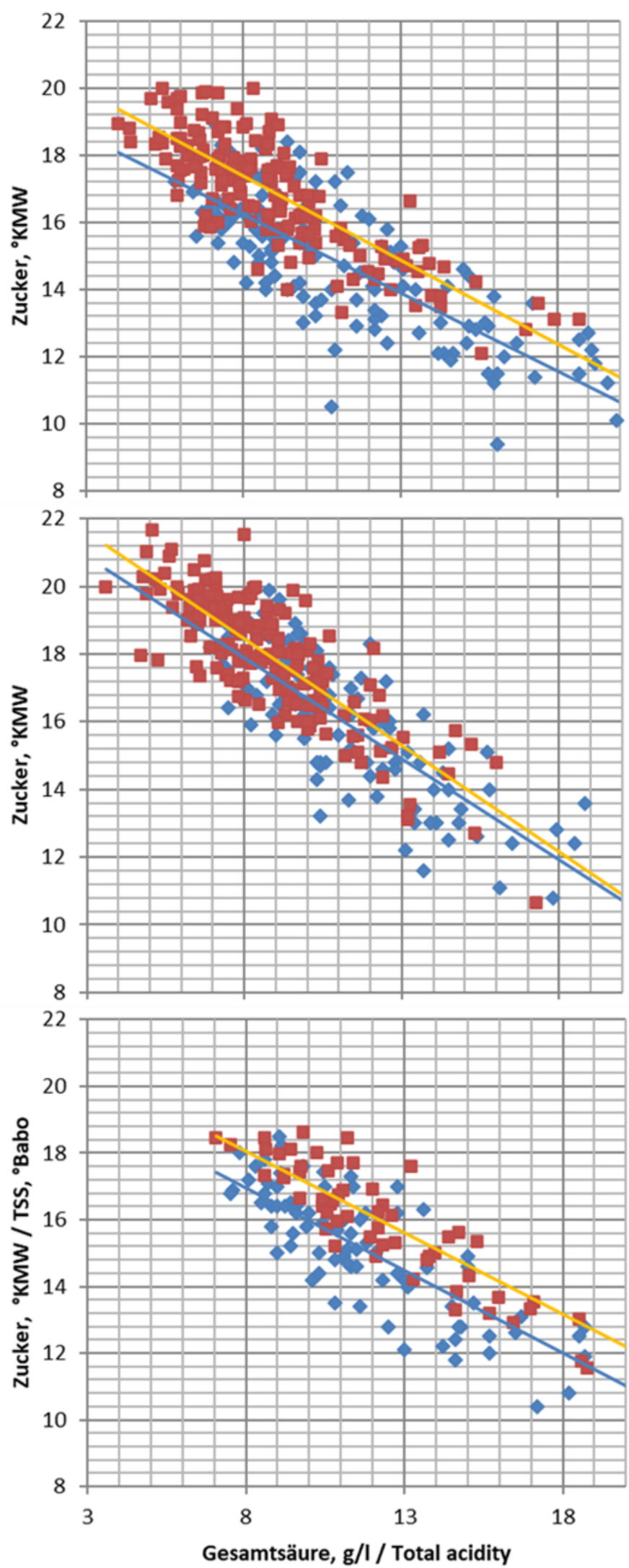

Weißburgunder /

Pinot blanc

Berg \& Terlan

1985-1999

- 2000-2019

— Linear (1985-1999)

— Linear (2000-2019)

\section{Blauburgunder /}

Pinot noir

Kastelbell \&

Mazzon

- 1985-1999

- 2000-2019

__ Linear (1985-1999)

L Linear (2000-2019)

\section{Chardonnay \\ Kurtinig}

- 1985-2004

- 2005-2019

— Linear (1985-2004)

L Linear (2005-2019) 

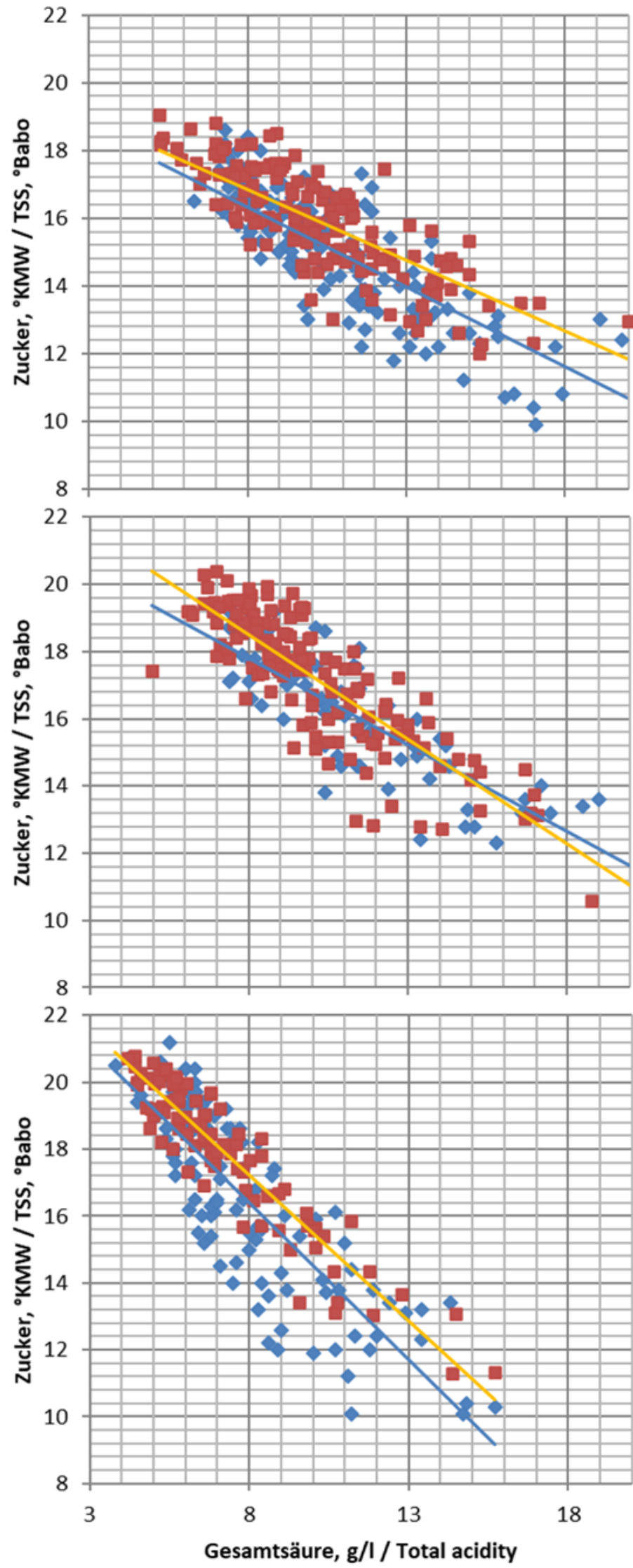

\section{Ruländer / Pinot gris \\ Salurn \& Kurtinig}

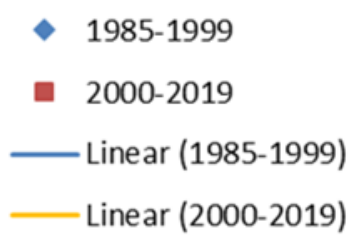

\section{Sauvignon}

Terlan \& Girlan

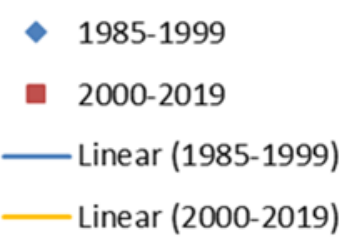

\section{Gewürztraminer Rungg}

- 1985-2002

- 2003-2019

— Linear (1985-2002)

_ Linear (2003-2019) 


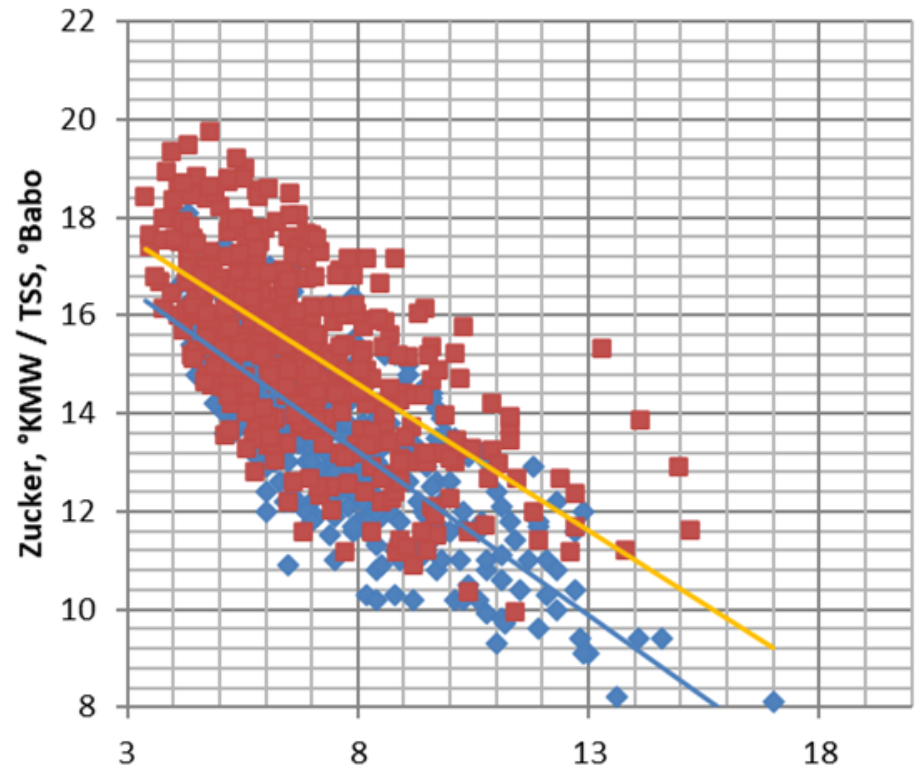

Gesamtsäure, g/l / Total acidity
Vernatsch

5 Lagen / 5 sites

- 1985-1999

- $2000-2019$

— Linear (1985-1999)

_Linear (2000 - 2019)

Abb. 5c. 
Abb. 6 a-c: Relationen der Most-Gesamtsäurewerte zu den pH-Werten der einzelnen Sorten und Anlagen. // Relations between must total acidity and $\mathrm{pH}$-valuse of the single varieties and sites.
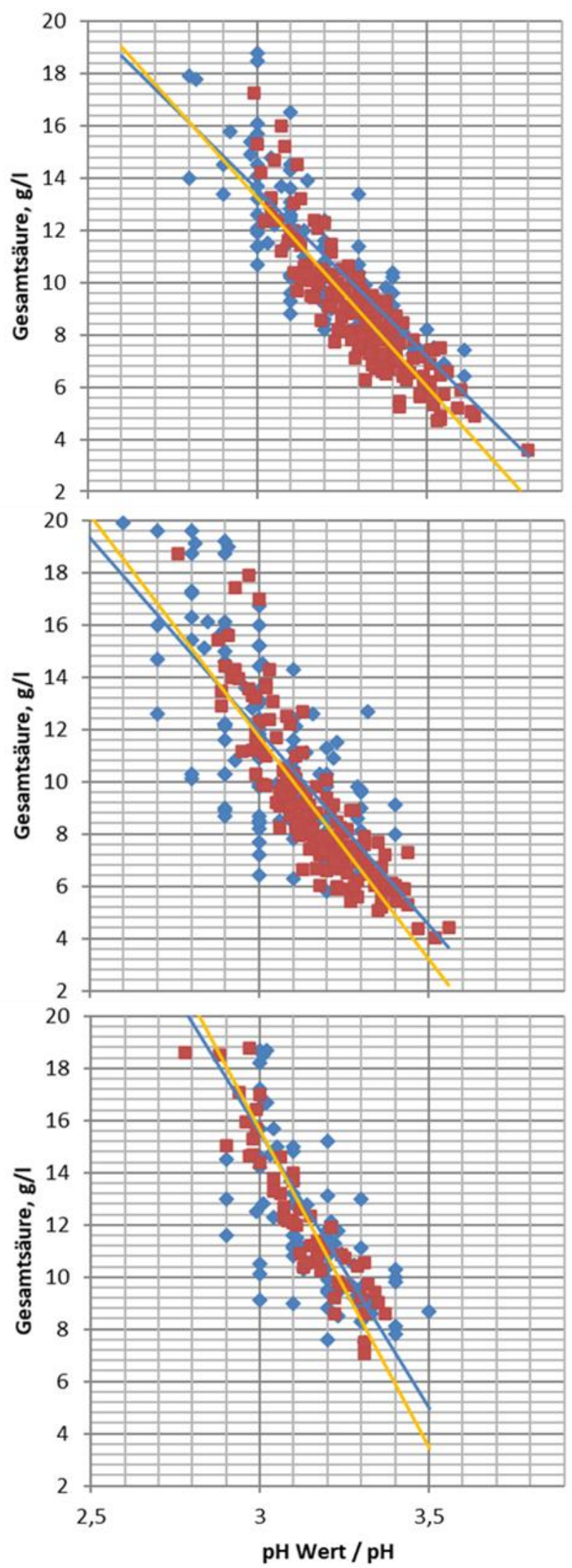

\author{
Blauburgunder / \\ Pinot noir \\ Kastelbell \& \\ Mazzon \\ - 1985-1999 \\ - 2000-2019 \\ — Linear (1985-1999) \\ _L Linear (2000-2019)
}

\section{Weißburgunder / \\ Pinot blanc}

Berg \& Terlan

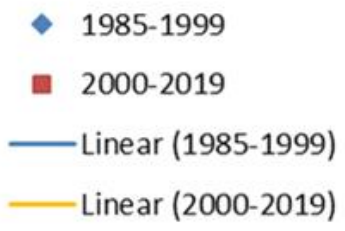

\section{Chardonnay \\ Kurtinig}

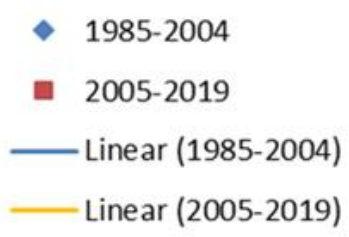

-1985-2004

- 2005-2019

_ Linear (2005-2019) 

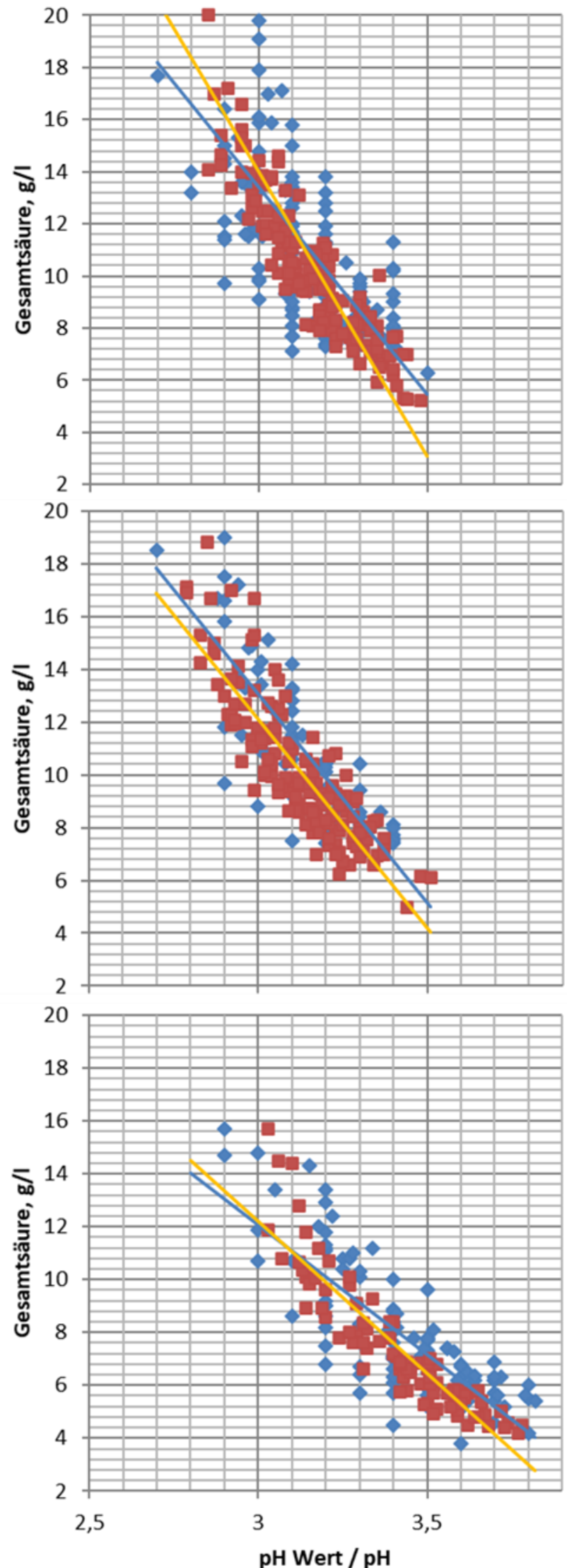

Ruländer / Pinot gris Salurn \& Kurtinig

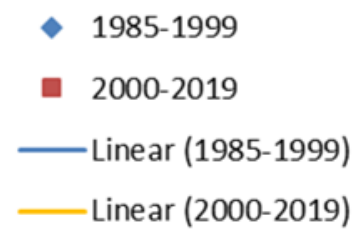

\section{Weißer Sauvignon} Terlan \& Girlan

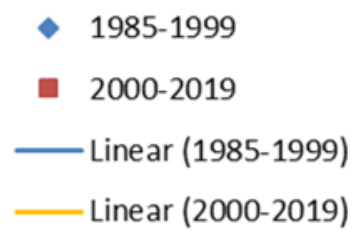

\section{Gewürztraminer Rungg}
- 1985-2002
- 2003-2019
— Linear (1985-2002)
— Linear (2003-2019) 


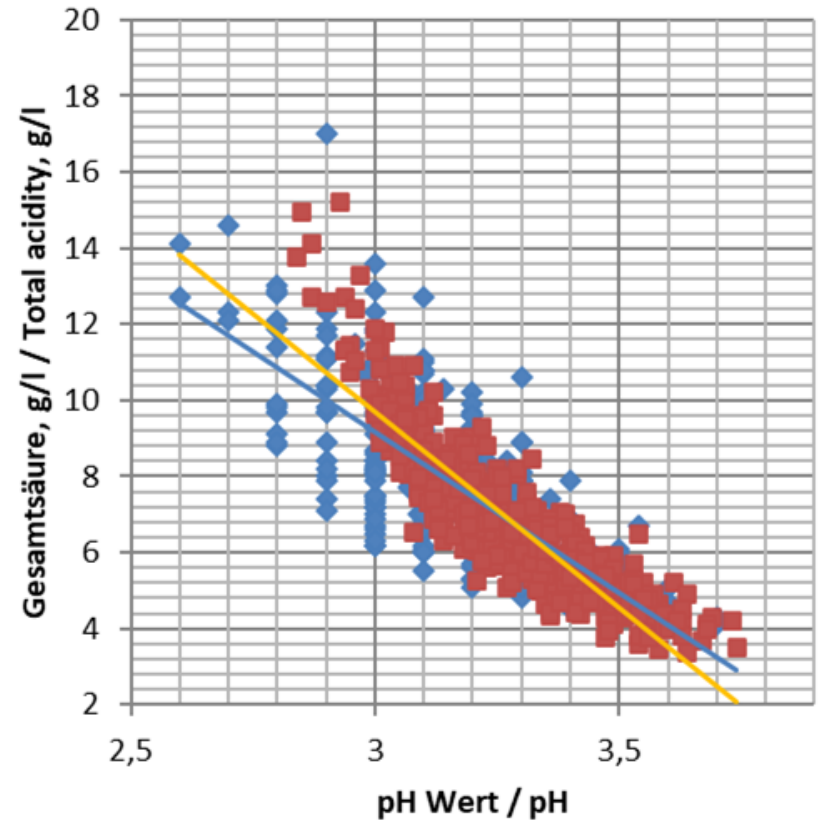

\section{Vernatsch \\ 5 Lagen / 5 sites}

1985-1999

- 2000-2019

L Linear (1985-1999)

Linear (2000-2019)

Abb. 6c.

\section{cc) (7) (9)}

Dieses Werk ist lizenziert unter einer Creative Commons Namensnennung-Nicht kommerziell 4.0 International Lizenz. Quest'opera è distribuita con Licenza Creative Commons Attribuzione - Non commerciale 4.0 Internazionale. This work is licensed under a Creative Commons Attribution-NonCommercial 4.0 International License.

Für alle Abbildungen und Tabellen ohne Nennung des Urhebers gilt: (c) Versuchszentrum Laimburg. Per tutte le immagini e tabelle senza menzione dell'artefice vale: (c) Centro di Sperimentazione Laimburg. For all figures and tables without mention of the originator applies: ( L Laimburg Research Centre. 